\title{
Polymerization of Phenylacetylene-Based Monodendrons with Alkoxy Peripheral Groups and Oxygen/Nitrogen Permeation Behavior of Their Membranes
}

\author{
Takashi Kaneko, ${ }^{1,2,3}$ Kazuaki Sato, ${ }^{2}$ Yoshihiko Uchiya, ${ }^{3}$ \\ Masahiro Teraguchi, ${ }^{1,2,3}$ and Toshiki Aoki ${ }^{1,2,3,4}$ \\ ${ }^{1}$ Center for Transdisciplinary Research, Niigata University, Ikarashi 2-8050, Niigata 950-2181, Japan \\ ${ }^{2}$ Department of Chemistry and Chemical Engineering, Faculty of Engineering, Niigata University, Ikarashi 2-8050, \\ Niigata 950-2181, Japan \\ ${ }^{3}$ Graduate School of Science and Technology, Niigata University, Ikarashi 2-8050, Niigata 950-2181, Japan \\ ${ }^{4}$ Venture Business Laboratory, Niigata University, Ikarashi 2-8050, Niigata 950-2181, Japan \\ Correspondence should be addressed to Takashi Kaneko, kanetaka@gs.niigata-u.ac.jp
}

Received 11 August 2011; Accepted 28 November 2011

Academic Editor: Eri Yoshida

Copyright (C) 2012 Takashi Kaneko et al. This is an open access article distributed under the Creative Commons Attribution License, which permits unrestricted use, distribution, and reproduction in any medium, provided the original work is properly cited.

\begin{abstract}
Monodendron monomers with alkoxy peripheral groups were synthesized, and the focal point of monodendrons, terminal acetylene, was polymerized with rhodium catalyst to yield corresponding polydendrons with a high molecular weight. The polydendrons were soluble in common organic solvents and readily formed membranes. Oxygen permselectivity was improved in the polydendrons with a space-persistent dendritic crowd. It was found that the well-defined dendritic and rod-like structure of the polydendrons was useful for permselective membrane.
\end{abstract}

\section{Introduction}

The chemical structure and/or morphology of polymers play an important role for the permselectivity of nonporous membranes. Recently, dendrimers have attracted much attention in organic and polymer chemistry due to their novel properties or functions, which are based on their specific shapes and precisely defined three-dimensional structures $[1-3]$. Dendrimers are characterized by a regularlyand fractally-branched treelike architecture. This feature provides a nanoscale homogeneous space in the dendrimers particularly suited for rigid molecular architectures. Additionally, the center and periphery of dendrimer molecule can be modified by substituent groups, which may aid molecular recognition [4].

We have previously synthesized monodendron monomers consisting of $m$-linked phenyleneethynylene repeating unit with trimethylsilyl peripheral groups and oligo(dimethylsiloxane) chains and obtained the corresponding polydendrons by polymerization of the monomers using a rhodium $(\mathrm{Rh})$ catalyst $[5,6]$. The polydendrons were mechanically stable and excellent for preparing selfsupporting membranes. The first-generation polydendron (polyTMS1H) membrane showed an oxygen permselectivity higher than that of the corresponding zero-generation poly(phenylacetylene) derivative (polyTMSOH). In this study, we synthesized polydendrons with alkoxy peripheral groups as shown in Scheme 1 because alkyl domain that would have better gas barrier property than that of oligo(dimethylsiloxane) domain would be suitable to study the effect of dendritic domain on the gas permselectivity. We examined the oxygen permselectivity of their membranes in relation to their chemical structures.

\section{Experimental}

2.1. Materials. 3,5-Dibromo-1-(3-hydroxyl-3-methylbutynyl)benzene was synthesized as previously described [7]. 

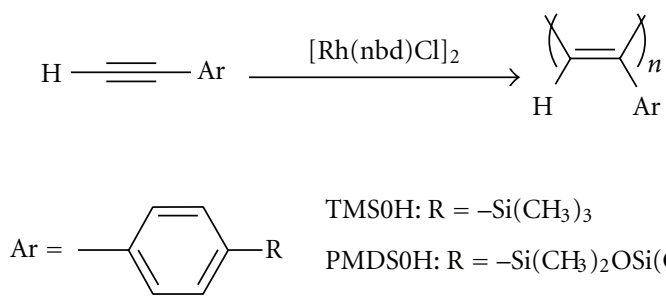

TMSOH: $\mathrm{R}=-\mathrm{Si}\left(\mathrm{CH}_{3}\right)_{3}$

PMDSOH: $\mathrm{R}=-\mathrm{Si}\left(\mathrm{CH}_{3}\right)_{2} \mathrm{OSi}\left(\mathrm{CH}_{3}\right)_{3}$ NMTSOH: $\mathrm{R}=-\left[\mathrm{Si}\left(\mathrm{CH}_{3}\right)_{2} \mathrm{O}\right]_{3} \mathrm{Si}\left(\mathrm{CH}_{3}\right)_{3}$ Hex0H: $\mathrm{R}=-\left(\mathrm{CH}_{2}\right)_{5} \mathrm{CH}_{3}$
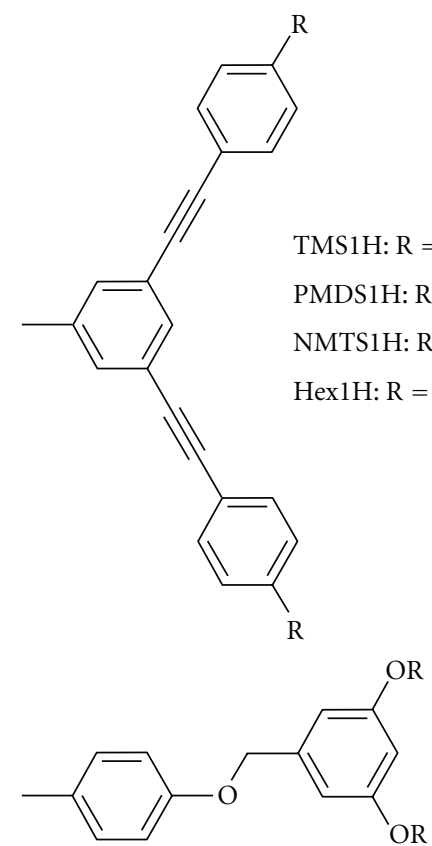

MGOPA: $\mathrm{R}=\mathrm{CH}_{3}$

HGOPA: $\mathrm{R}=\mathrm{C}_{6} \mathrm{H}_{13}$

DGOPA: $\mathrm{R}=\mathrm{C}_{12} \mathrm{H}_{25}$

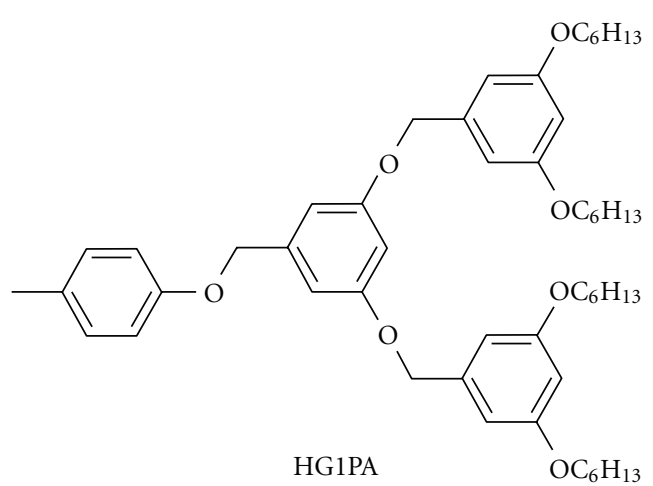

Scheme 1

3,5-Dimethoxybenzyl chloride [8], 3,5-dihexyloxybenzyl alcohol [9], 3,5-didodecyloxybenzyl alcohol [10], 3,5bis(3,5-dihexyloxybenzyloxy)benzyl alcohol [11], and (4hexyloxyphenyl)acetylene (Hex0H) [12] were synthesized according to the literature procedures. (Bicyclo [2.2.1] hepta2,5-diene)chlororhodium(I) dimer catalyst ( $\left.[\mathrm{Rh}(\mathrm{nbd}) \mathrm{Cl}]_{2}\right)$ (Aldrich Co.), bis(triphenylphosphine)palladium(II) chloride (Aldrich Co.), and $n$-butyllithium (Kanto Chemical Co., Inc., $1.6 \mathrm{M}$ in hexane) were used without further purification. Other conventional reagents were used as received or purified by conventional method.
2.2. Synthesis of Monomers. The general procedure of 3,5-dialkoxybenzyl chloride is as follows. A solution of 3,5-dialkoxybenzyl alcohol $(5 \mathrm{mmol}), \mathrm{DMF}(0.5 \mathrm{~mL})$, and thionyl chloride $(10 \mathrm{mmol})$ in chloroform $(25 \mathrm{~mL})$ was stirred at room temperature for $2-12 \mathrm{~h}$. The excess thionyl chloride and solvents were evaporated under reduced pressure, and then the residue was dissolved in diethyl ether. The ether solution was washed with water, then dried over anhydrous sodium sulfate, filtered, and evaporated. Then, 3,5-dialkoxybenzyl chloride was obtained without further purification.

3,5-Dihexyloxybenzyl Chloride. Yield 94\%. IR $\left(\mathrm{KBr} ; \mathrm{cm}^{-1}\right)$ : 2872-2936 (C-H). ${ }^{1} \mathrm{H}$ NMR $\left(\mathrm{CDCl}_{3}, 270 \mathrm{MHz} ; \mathrm{ppm}\right): \delta$ $0.91\left(\mathrm{t}, 6 \mathrm{H}, J=6.8 \mathrm{~Hz}, \mathrm{CH}_{3}\right), 1.33\left(\mathrm{~m}, 8 \mathrm{H}, \mathrm{CH}_{2}\right), 1.44$ $\left(\mathrm{m}, 4 \mathrm{H}, \mathrm{CH}_{2}\right), 1.77\left(\mathrm{~m}, 4 \mathrm{H}, \mathrm{CH}_{2}\right), 3.93(\mathrm{t}, 4 \mathrm{H}, J=6.5 \mathrm{~Hz}$, $\mathrm{CH}_{2} \mathrm{O}$ ), 4.50 (s, $\left.2 \mathrm{H}, \mathrm{CH}_{2} \mathrm{Cl}\right), 6.39(\mathrm{t}, 1 \mathrm{H}, J=2.4 \mathrm{~Hz}, \mathrm{ArH})$, $6.51(\mathrm{~d}, 2 \mathrm{H}, J=2.4 \mathrm{~Hz}, \mathrm{ArH}) .{ }^{13} \mathrm{C} \mathrm{NMR}\left(\mathrm{CDCl}_{3} ; \mathrm{ppm}\right): \delta$ $14.10,22.66,25.68,29.07,31.55,46.46,68.07,101.17,106.78$, $139.16,160.26$.

3,5-Didodecyloxybenzyl Chloride. Yield 76\%. IR ( $\mathrm{KBr}$; $\left.\mathrm{cm}^{-1}\right)$ : $2856-2928(\mathrm{C}-\mathrm{H}) .{ }^{1} \mathrm{H}$ NMR $\left(\mathrm{CDCl}_{3}, 500 \mathrm{MHz}\right.$; ppm): $\delta 0.88\left(\mathrm{t}, 6 \mathrm{H}, J=6.5 \mathrm{~Hz}, \mathrm{CH}_{3}\right), 1.27\left(\mathrm{~m}, 32 \mathrm{H}, \mathrm{CH}_{2}\right)$, $1.44\left(\mathrm{~m}, 4 \mathrm{H}, \mathrm{CH}_{2}\right), 1.76\left(\mathrm{~m}, 4 \mathrm{H}, \mathrm{CH}_{2}\right), 3.93(\mathrm{t}, 4 \mathrm{H}, J=$ $\left.6.5 \mathrm{~Hz}, \mathrm{CH}_{2} \mathrm{O}\right), 4.50\left(\mathrm{~s}, 2 \mathrm{H}, \mathrm{CH}_{2} \mathrm{Cl}\right), 6.40(\mathrm{t}, 1 \mathrm{H}, J=2.5 \mathrm{~Hz}$, $\mathrm{ArH}), 6.51(\mathrm{~d}, 2 \mathrm{H}, J=2.5 \mathrm{~Hz}, \mathrm{ArH})$.

3,5-Bis(3,5-dihexyloxybenzyloxy)benzyl chloride. Yield 99\%. IR $\left(\mathrm{KBr} ; \mathrm{cm}^{-1}\right): 2868-2944(\mathrm{C}-\mathrm{H}) .{ }^{1} \mathrm{H}$ NMR $\left(\mathrm{CDCl}_{3}\right.$, $270 \mathrm{MHz} ; \mathrm{ppm}): \delta 0.90\left(\mathrm{t}, 12 \mathrm{H}, J=7.0 \mathrm{~Hz}, \mathrm{CH}_{3}\right), 1.34(\mathrm{~m}$, $\left.16 \mathrm{H}, \mathrm{CH}_{2}\right), 1.45\left(\mathrm{~m}, 8 \mathrm{H}, \mathrm{CH}_{2}\right), 1.76\left(\mathrm{~m}, 8 \mathrm{H}, \mathrm{CH}_{2}\right), 3.93(\mathrm{t}$, $\left.8 \mathrm{H}, J=6.5 \mathrm{~Hz}, \mathrm{CH}_{2} \mathrm{O}\right), 4.50\left(\mathrm{~s}, 2 \mathrm{H}, \mathrm{CH}_{2} \mathrm{Cl}\right), 4.94(\mathrm{~s}, 4 \mathrm{H}$, $\left.\mathrm{CH}_{2} \mathrm{O}\right), 6.40(\mathrm{t}, 2 \mathrm{H}, J=2.2 \mathrm{~Hz}, \mathrm{ArH}), 6.51(\mathrm{~d}, 4 \mathrm{H}, J=2.2 \mathrm{~Hz}$, $\mathrm{ArH}), 6.55(\mathrm{t}, 1 \mathrm{H}, J=2.2 \mathrm{~Hz}, \mathrm{ArH}), 6.62(\mathrm{~d}, 2 \mathrm{H}, J=2.2 \mathrm{~Hz}$, $\mathrm{ArH}) .{ }^{13} \mathrm{C}$ NMR $\left(\mathrm{CDCl}_{3} ; \mathrm{ppm}\right): \delta 14.14,22.68,25.79,29.27$, $31.64,46.35,68.04,70.15,100.75,101.99,105.62,107.53$, $138.62,139.34,159.88,160.33$.

4-Bromo-1-(3,5-dimethoxybenzyloxy)benzene. A mixture of 4-bromophenol (13 g, $74 \mathrm{mmol}$ ), 3,5-dimethoxybenzyl chloride $(15 \mathrm{~g}, 81 \mathrm{mmol}), \mathrm{K}_{2} \mathrm{CO}_{3}(22 \mathrm{~g}, 0.16 \mathrm{~mol})$, and 18-crown$6(3.0 \mathrm{~g}, 11 \mathrm{mmol})$ in acetone $(200 \mathrm{~mL})$ was refluxed for $18 \mathrm{~h}$. After removal of the solvent, the residue was extracted with dichloromethane. The organic layer was washed with brine, then dried over anhydrous sodium sulfate, filtered, and evaporated. The crude product was purified by silicagel column separation with dichloromethane/hexane (1/1 $\mathrm{v} / \mathrm{v})$ as an eluent to give 4-bromo-1-(3,5-dimethoxybenzyloxy)benzene ( $19 \mathrm{~g}, 58 \mathrm{mmol})$. Yield $78 \%$; $\mathrm{mp} 75^{\circ} \mathrm{C}$. TLC (dichloromethane/hexane $(1 / 1 \mathrm{v} / \mathrm{v})): R_{f}=0.14$. IR $(\mathrm{KBr}$; $\left.\mathrm{cm}^{-1}\right)$ : 2844-2944 (C-H). ${ }^{1} \mathrm{H}$ NMR $\left(\mathrm{CDCl}_{3}, 270 \mathrm{MHz}\right.$; ppm): $\delta 3.77\left(\mathrm{~s}, 6 \mathrm{H}, \mathrm{CH}_{3}\right), 4.96\left(\mathrm{~s}, 2 \mathrm{H}, \mathrm{CH}_{2}\right), 6.41(\mathrm{t}, 1 \mathrm{H}$, $J=2.4 \mathrm{~Hz}, \mathrm{ArH}), 6.55(\mathrm{~d}, 2 \mathrm{H}, J=2.4 \mathrm{~Hz}, \mathrm{ArH}), 6.83(\mathrm{~d}, 2 \mathrm{H}$, $J=8.9 \mathrm{~Hz}, \mathrm{ArH}), 7.36(\mathrm{~d}, 2 \mathrm{H}, J=8.9 \mathrm{~Hz}, \mathrm{ArH}) .{ }^{13} \mathrm{C} \mathrm{NMR}$ $\left(\mathrm{CDCl}_{3} ; \mathrm{ppm}\right): \delta 55.36,70.10,99.80,105.05,113.04,116.58$, $132.13,138.78,157.57,160.85$. 
1-(3,5-Dimethoxybenzyloxy)-4-(3-hydroxyl-3-methylbutynyl) benzene. 2-Methyl-3-butyn-2-ol (10 mL, $0.10 \mathrm{~mol})$ was added to a triethylamine solution $(110 \mathrm{~mL})$ of 4 -bromo1-(3,5-dimethoxybenzyloxy)benzene ( $18 \mathrm{~g}, 55 \mathrm{mmol})$, bis (triphenylphosphine)palladium (II) chloride $(0.14 \mathrm{~g}$, $0.2 \mathrm{mmol})$, triphenylphosphine $(0.72 \mathrm{~g}, 2.7 \mathrm{mmol})$, and copper (I) iodide $(0.34 \mathrm{~g}, 1.8 \mathrm{mmol})$ under a nitrogen atmosphere. The solution was stirred for $18 \mathrm{~h}$ at $90^{\circ} \mathrm{C}$. After cooling, the mixture was treated with aqueous $4 \mathrm{~N} \mathrm{HCl}$, extracted with dichloromethane, and washed with brine. The organic layer was dried over anhydrous sodium sulfate, and the solvent was removed under reduced pressure. The crude product was purified by silica gel column separation with dichloromethane as an eluent to give 1-(3,5dimethoxybenzyloxy)-4-(3-hydroxyl-3-methylbutynyl)benzene $(5.5 \mathrm{~g}, 17 \mathrm{mmol})$. Yield $31 \%$. TLC (dichloromethane): $R_{f}=0.15$. IR $\left(\mathrm{KBr} ; \mathrm{cm}^{-1}\right): 3324(-\mathrm{OH}), 2848-2988(\mathrm{C}-\mathrm{H})$, $2228(\mathrm{C} \equiv \mathrm{C}) .{ }^{1} \mathrm{H}$ NMR $\left(\mathrm{CDCl}_{3}, 270 \mathrm{MHz} ; \mathrm{ppm}\right): \delta 1.61$ (s, $\left.6 \mathrm{H}, \mathrm{CH}_{3}\right), 2.11(\mathrm{~s}, 1 \mathrm{H},-\mathrm{OH}), 3.79\left(\mathrm{~s}, 6 \mathrm{H}, \mathrm{CH}_{3}\right), 4.99(\mathrm{~s}, 2 \mathrm{H}$, $\left.\mathrm{CH}_{2}\right), 6.41(\mathrm{t}, 1 \mathrm{H}, J=2.2 \mathrm{~Hz}, \mathrm{ArH}), 6.56(\mathrm{~d}, 2 \mathrm{H}, J=2.2 \mathrm{~Hz}$, $\operatorname{ArH}), 6.88(\mathrm{~d}, 2 \mathrm{H}, J=8.9 \mathrm{~Hz}, \mathrm{ArH}), 7.34(\mathrm{~d}, 2 \mathrm{H}, J=8.9 \mathrm{~Hz}$, $\mathrm{ArH}) .{ }^{13} \mathrm{C} \mathrm{NMR}\left(\mathrm{CDCl}_{3} ; \mathrm{ppm}\right): \delta 31.61,55.36,65.61,69.89$, $81.89,92.45,99.82,105.08,114.69,115.03,132.94,138.81$, $158.44,160.82$.

[4-(3,5-Dimethoxybenzyloxy)phenyl]acetylene (MGOPA). Sodium hydride $(2.0 \mathrm{~g}, 50 \mathrm{mmol})$ was added to a toluene solution (65 mL) of 1-(3,5-dimethoxybenzyloxy)-4-(3hydroxyl-3-methylbutynyl)benzene $(5.5 \mathrm{~g}, 17 \mathrm{mmol})$. The solution was refluxed for $2 \mathrm{~h}$ under a nitrogen atmosphere. After cooling, the mixture was treated with water, extracted with dichloromethane, and washed with brine. The organic layer was dried over anhydrous sodium sulfate, and the solvent was removed under reduced pressure. The crude product was purified by silica gel column separation with dichloromethane as an eluent to give MG0PA (1.1 g, $4.3 \mathrm{mmol}$ ). Yield $25 \% ; \mathrm{mp} 60^{\circ} \mathrm{C}$. TLC (hexane): $R_{f}=0.75$. IR $\left(\mathrm{KBr} ; \mathrm{cm}^{-1}\right): 3296$ (三C-H), 2848-2988 (C-H), 2112 $(\mathrm{C} \equiv \mathrm{C}) .{ }^{1} \mathrm{H}$ NMR $\left(\mathrm{CDCl}_{3}, 270 \mathrm{MHz} ; \mathrm{ppm}\right): \delta 3.00(\mathrm{~s}, 1 \mathrm{H}$, $\mathrm{C} \equiv \mathrm{C}-\mathrm{H}), 3.78\left(\mathrm{~s}, 6 \mathrm{H}, \mathrm{CH}_{3}\right), 4.98\left(\mathrm{~s}, 2 \mathrm{H}, \mathrm{CH}_{2}\right), 6.40(\mathrm{t}, 1 \mathrm{H}$, $J=1.9 \mathrm{~Hz}, \operatorname{ArH}), 6.55(\mathrm{~d}, 2 \mathrm{H}, J=1.9 \mathrm{~Hz}, \operatorname{ArH}), 6.89(\mathrm{~d}, 2 \mathrm{H}$, $J=8.9 \mathrm{~Hz}, \operatorname{ArH}), 7.41(\mathrm{~d}, 2 \mathrm{H}, J=8.9 \mathrm{~Hz}, \mathrm{ArH}) .{ }^{13} \mathrm{C} \mathrm{NMR}$ $\left(\mathrm{CDCl}_{3} ; \mathrm{ppm}\right): \delta 55.32,69.87,75.96,83.53,99.80,105.04$, 114.31, 114.70, 133.43, 138.71, 158.79, 160.81.

4-Bromo-1-(3,5-didodecyloxybenzyloxy)benzene. A mixture of 4-bromophenol (1.9 g, $11 \mathrm{mmol}), 3,5$-didodecyloxybenzyl chloride $(6.0 \mathrm{~g}, 12 \mathrm{mmol}), \mathrm{K}_{2} \mathrm{CO}_{3} \quad(3.3 \mathrm{~g}, 24 \mathrm{mmol})$, and 18 -crown-6 $(3.2 \mathrm{~g}, 12 \mathrm{mmol})$ in acetone $(60 \mathrm{~mL})$ was refluxed for $87 \mathrm{~h}$. The mixture was worked up in the same manner as described above, and the crude product was purified by silica gel column separation with dichloromethane/hexane $(1 / 2 \mathrm{v} / \mathrm{v})$ as an eluent to give 4-bromo-1-(3,5-didodecyloxybenzyloxy)benzene (5.3 g, $8.3 \mathrm{mmol}$ ). Yield $76 \%$. TLC (dichloromethane/hexane $(1 / 2$ $\mathrm{v} / \mathrm{v})): R_{f}=0.90 . \mathrm{IR}\left(\mathrm{KBr} ; \mathrm{cm}^{-1}\right): 2856-2928(\mathrm{C}-\mathrm{H}) .{ }^{1} \mathrm{H}$ NMR $\left(\mathrm{CDCl}_{3}, 500 \mathrm{MHz} ; \mathrm{ppm}\right): \delta 0.88(\mathrm{t}, 6 \mathrm{H}, J=6.8 \mathrm{~Hz}$, $\left.\mathrm{CH}_{3}\right), 1.26\left(\mathrm{~m}, 32 \mathrm{H}, \mathrm{CH}_{2}\right), 1.43\left(\mathrm{~m}, 4 \mathrm{H}, \mathrm{CH}_{2}\right), 1.76(\mathrm{~m}, 4 \mathrm{H}$,
$\left.\mathrm{CH}_{2}\right), 3.92\left(\mathrm{t}, 4 \mathrm{H}, J=6.8 \mathrm{~Hz}, \mathrm{CH}_{2} \mathrm{O}\right), 4.95\left(\mathrm{~s}, 2 \mathrm{H}, \mathrm{CH}_{2} \mathrm{O}\right)$, $6.40(\mathrm{t}, 1 \mathrm{H}, J=2.3 \mathrm{~Hz}, \mathrm{ArH}), 6.52(\mathrm{~d}, 2 \mathrm{H}, J=2.3 \mathrm{~Hz}, \operatorname{ArH})$, $6.84(\mathrm{~d}, 2 \mathrm{H}, J=8.8 \mathrm{~Hz}, \mathrm{ArH}), 7.36(\mathrm{~d}, 2 \mathrm{H}, J=8.8 \mathrm{~Hz}, \mathrm{ArH})$.

1-(3,5-Didodecyloxybenzyloxy)-4-(3-hydroxyl-3-methylbutynyl)benzene. 2-Methyl-3-butyn-2-ol (1.2 mL, $12 \mathrm{mmol})$ was added to a triethylamine solution $(12 \mathrm{~mL})$ of 4-bromo1-(3,5-didodecyloxybenzyloxy)benzene $(4.0 \mathrm{~g}, 6.3 \mathrm{mmol})$, bis(triphenylphosphine)palladium (II) chloride (1.6 mg, $0.0022 \mathrm{mmol})$, triphenylphosphine $(2.8 \mathrm{mg}, 0.010 \mathrm{mmol})$, and copper (I) iodide $(1.5 \mathrm{mg}, 0.0077 \mathrm{mmol})$ under a nitrogen atmosphere. The solution was stirred for $24 \mathrm{~h}$ at $90^{\circ} \mathrm{C}$. The mixture was worked up in the same manner as described above, and the crude product was purified by silica gel column separation with dichloromethane/hexane $(1 / 2$ $\mathrm{v} / \mathrm{v})$ as an eluent to give 1-(3,5-didodecyloxybenzyloxy)4-(3-hydroxyl-3-methylbutynyl)benzene (1.6 g, $2.5 \mathrm{mmol})$. Yield 40\%. IR $\left(\mathrm{KBr} ; \mathrm{cm}^{-1}\right): 3324(-\mathrm{OH}), 2856-2928(\mathrm{C}-\mathrm{H})$, $2240(\mathrm{C} \equiv \mathrm{C}) .{ }^{1} \mathrm{H}$ NMR $\left(\mathrm{CDCl}_{3}, 500 \mathrm{MHz} ; \mathrm{ppm}\right): \delta 0.88(\mathrm{t}$, $\left.6 \mathrm{H}, J=6.5 \mathrm{~Hz}, \mathrm{CH}_{3}\right), 1.26\left(\mathrm{~m}, 32 \mathrm{H}, \mathrm{CH}_{2}\right), 1.42(\mathrm{~m}, 4 \mathrm{H}$, $\left.\mathrm{CH}_{2}\right), 1.60\left(\mathrm{~s}, 6 \mathrm{H}, \mathrm{CH}_{3}\right), 1.76\left(\mathrm{~m}, 4 \mathrm{H}, \mathrm{CH}_{2}\right), 2.04(\mathrm{~s}, 1 \mathrm{H}$, $-\mathrm{OH}), 3.92\left(\mathrm{t}, 4 \mathrm{H}, J=6.5 \mathrm{~Hz}, \mathrm{CH}_{2} \mathrm{O}\right), 4.98\left(\mathrm{~s}, 2 \mathrm{H}, \mathrm{CH}_{2} \mathrm{O}\right)$, $6.40(\mathrm{t}, 1 \mathrm{H}, J=2.0 \mathrm{~Hz}, \mathrm{ArH}), 6.53(\mathrm{~d}, 2 \mathrm{H}, J=2.0 \mathrm{~Hz}, \mathrm{ArH})$, $6.88(\mathrm{~d}, 2 \mathrm{H}, J=8.5 \mathrm{~Hz}, \mathrm{ArH}), 7.34(\mathrm{~d}, 2 \mathrm{H}, J=8.5 \mathrm{~Hz}, \mathrm{ArH})$. ${ }^{13} \mathrm{C} \mathrm{NMR}\left(\mathrm{CDCl}_{3} ; \mathrm{ppm}\right): \delta 14.18,22.67,26.03,29.23,29.34$, $29.38,29.56,29.58,29.62,29.65,31.56,31.91,65.63,68.07$, $70.01,81.96,92.44,100.79,105.60,114.80,115.05,133.04$, $138.75,158.70,160.53$.

[4-(3,5-Didodecyloxybenzyloxy)phenyl]acetylene (DGOPA). Catalytic amounts of sodium hydride were added to a toluene solution (12 mL) of 1-(3,5-didodecyloxybenzyloxy)-4-(3hydroxyl-3-methylbutynyl)benzene $(0.90 \mathrm{~g}, 1.4 \mathrm{mmol})$. The solution was refluxed for $3 \mathrm{~h}$ under a nitrogen atmosphere. The mixture was worked up in the same manner as described above, and the crude product was purified by silica gel column separation with dichloromethane/hexane $(1 / 1 \mathrm{v} / \mathrm{v})$ and hexane as an eluent to give DG0PA $(0.45 \mathrm{~g}$, $0.78 \mathrm{mmol})$. Yield 55\%. TLC (dichloromethane/hexane $(1 / 1$ $\mathrm{v} / \mathrm{v})): R_{f}=0.71 . \mathrm{IR}\left(\mathrm{KBr} ; \mathrm{cm}^{-1}\right): 3328(\equiv \mathrm{C}-\mathrm{H}), 2856-2924$ $(\mathrm{C}-\mathrm{H}), 2112(\mathrm{C} \equiv \mathrm{C}) .{ }^{1} \mathrm{H}$ NMR $\left(\mathrm{CDCl}_{3}, 500 \mathrm{MHz} ; \mathrm{ppm}\right)$ : $\delta 0.88\left(\mathrm{t}, 6 \mathrm{H}, J=7.0 \mathrm{~Hz}, \mathrm{CH}_{3}\right), 1.26\left(\mathrm{~m}, 32 \mathrm{H}, \mathrm{CH}_{2}\right), 1.43$ $\left(\mathrm{m}, 4 \mathrm{H}, \mathrm{CH}_{2}\right), 1.76\left(\mathrm{~m}, 4 \mathrm{H}, \mathrm{CH}_{2}\right), 2.99(\mathrm{~s}, 1 \mathrm{H}, \mathrm{C} \equiv \mathrm{C}-\mathrm{H})$, $3.92\left(\mathrm{t}, 4 \mathrm{H}, J=6.5 \mathrm{~Hz}, \mathrm{CH}_{2} \mathrm{O}\right), 4.98\left(\mathrm{~s}, 2 \mathrm{H}, \mathrm{CH}_{2} \mathrm{O}\right), 6.40(\mathrm{t}$, $1 \mathrm{H}, J=2.0 \mathrm{~Hz}, \mathrm{ArH}), 6.53(\mathrm{~d}, 2 \mathrm{H}, J=2.0 \mathrm{~Hz}, \mathrm{ArH}), 6.90$ $(\mathrm{d}, 2 \mathrm{H}, J=8.5 \mathrm{~Hz}, \mathrm{ArH}), 7.41(\mathrm{~d}, 2 \mathrm{H}, J=8.5 \mathrm{~Hz}, \mathrm{ArH}) .{ }^{13} \mathrm{C}$ NMR $\left(\mathrm{CDCl}_{3} ; \mathrm{ppm}\right): \delta 14.11,22.68,26.03,29.23,29.34$, $29.38,29.56,29.59,29.63,29.65,31.91,68.06,70.02,75.81$, 83.60, 100.78, 105.56, 114.38, 114.83, 133.56, 138.66, 159.06, 160.53 .

4-Iodo-1-(3,5-dihexyloxybenzyloxy)benzene. A mixture of 4-iodophenol (18 g, $80 \mathrm{mmol}), 3,5$-dihexyloxybenzyl chloride $(29 \mathrm{~g}, \quad 88 \mathrm{mmol}), \mathrm{K}_{2} \mathrm{CO}_{3} \quad(24 \mathrm{~g}, 18 \mathrm{mmol})$, and 18 -crown-6 $(23 \mathrm{~g}, 88 \mathrm{mmol})$ in acetone $(530 \mathrm{~mL})$ was refluxed for $8 \mathrm{~h}$. The mixture was worked up in the same manner as described above, and the crude product was purified by silica gel column separation with 
dichloromethane/hexane $(1 / 3 \mathrm{v} / \mathrm{v})$ as an eluent to give 4iodo-1-(3,5-dihexyloxybenzyloxy)benzene (37 g, $73 \mathrm{mmol})$. Yield 91\%. TLC (dichloromethane/hexane $(1 / 3 \mathrm{v} / \mathrm{v})): R_{f}=$ 0.40. IR (KBr; cm $\left.{ }^{-1}\right): 2872-2936(\mathrm{C}-\mathrm{H}) .{ }^{1} \mathrm{H} \mathrm{NMR}\left(\mathrm{CDCl}_{3}\right.$, $270 \mathrm{MHz} ; \mathrm{ppm}): \delta 0.90\left(\mathrm{t}, 6 \mathrm{H}, J=6.8 \mathrm{~Hz}, \mathrm{CH}_{3}\right), 1.32(\mathrm{~m}$, $\left.8 \mathrm{H}, \mathrm{CH}_{2}\right), 1.44\left(\mathrm{~m}, 4 \mathrm{H}, \mathrm{CH}_{2}\right), 1.76\left(\mathrm{~m}, 4 \mathrm{H}, \mathrm{CH}_{2}\right), 3.92(\mathrm{t}$, $\left.4 \mathrm{H}, \mathrm{J}=6.8 \mathrm{~Hz}, \mathrm{CH}_{2} \mathrm{O}\right), 4.94\left(\mathrm{~s}, 2 \mathrm{H}, \mathrm{CH}_{2} \mathrm{O}\right), 6.39(\mathrm{t}, 1 \mathrm{H}, J$ $=2.2 \mathrm{~Hz}, \operatorname{ArH}), 6.52(\mathrm{~d}, 2 \mathrm{H}, J=2.2 \mathrm{~Hz}, \operatorname{ArH}), 6.73(\mathrm{~d}, 2 \mathrm{H}$, $J=9.3 \mathrm{~Hz}, \mathrm{ArH}), 7.53(\mathrm{~d}, 2 \mathrm{H}, J=9.3 \mathrm{~Hz}, \mathrm{ArH}) .{ }^{13} \mathrm{C} \mathrm{NMR}$ $\left(\mathrm{CDCl}_{3} ; \mathrm{ppm}\right): \delta 14.13,22.68,25.79,29.27,31.64,68.07$, $70.08,82.99,100.72,105.48,117.22,138.07,138.57,158.43$, 160.39 .

1-[4-(3,5-Dihexyloxybenzyloxy)phenyl]-2-trimethylsilylacetylene. Trimethylsilylacetylene $(19 \mathrm{~mL}, 0.14 \mathrm{~mol})$ was added to a triethylamine solution $(140 \mathrm{~mL})$ of 4 -iodo1-(3,5-dihexyloxybenzyloxy)benzene (36 g, $70 \mathrm{mmol})$, bis(triphenylphosphine)palladium (II) chloride (84 mg, $0.12 \mathrm{mmol})$, triphenylphosphine $(87 \mathrm{mg}, 0.33 \mathrm{mmol}$ ), and copper (I) iodide ( $46 \mathrm{mg}, 0.24 \mathrm{mmol}$ ) under a nitrogen atmosphere. The solution was stirred for $10 \mathrm{~h}$ at $60^{\circ} \mathrm{C}$. The mixture was worked up in the same manner as described above, and the crude product was purified by silica gel column separation with dichloromethane/hexane $(1 / 2 \mathrm{v} / \mathrm{v})$ as an eluent to give 1-[4-(3,5-dihexyloxybenzyloxy)phenyl]2-trimethylsilylacetylene $(25 \mathrm{~g}, 53 \mathrm{mmol})$. Yield $76 \%$. IR $\left(\mathrm{KBr} ; \mathrm{cm}^{-1}\right)$ : 2872-2964 (C-H), 2160 (C三C). ${ }^{1} \mathrm{H}$ NMR $\left(\mathrm{CDCl}_{3}, 270 \mathrm{MHz} ; \mathrm{ppm}\right): \delta 0.24\left(\mathrm{~s}, 9 \mathrm{H}, \mathrm{Si}\left(\mathrm{CH}_{3}\right)_{3}\right), 0.91(\mathrm{t}$, $\left.6 \mathrm{H}, J=6.8 \mathrm{~Hz}, \mathrm{CH}_{3}\right), 1.33\left(\mathrm{~m}, 8 \mathrm{H}, \mathrm{CH}_{2}\right), 1.45\left(\mathrm{~m}, 4 \mathrm{H}, \mathrm{CH}_{2}\right)$, $1.76\left(\mathrm{~m}, 4 \mathrm{H}, \mathrm{CH}_{2}\right), 3.93\left(\mathrm{t}, 4 \mathrm{H}, \mathrm{J}=6.5 \mathrm{~Hz}, \mathrm{CH}_{2} \mathrm{O}\right), 4.98(\mathrm{~s}$, $\left.2 \mathrm{H}, \mathrm{CH}_{2} \mathrm{O}\right), 6.40(\mathrm{t}, 1 \mathrm{H}, J=2.4 \mathrm{~Hz}, \mathrm{ArH}), 6.53(\mathrm{~d}, 2 \mathrm{H}, J=$ $2.4 \mathrm{~Hz}, \mathrm{ArH}), 6.88$ (d, 2H, $J=8.9 \mathrm{~Hz}, \mathrm{ArH}), 7.39$ (d, 2H, $J=$ $8.9 \mathrm{~Hz}, \mathrm{ArH}) .{ }^{13} \mathrm{C} \mathrm{NMR}\left(\mathrm{CDCl}_{3} ; \mathrm{ppm}\right): \delta 0.17,14.14,22.68$, $25.79,29.26,31.64,68.05,69.97,92.46,100.71,105.07$, $105.49,114.64,115.39,133.33,138.58,158.69,160.35$.

[4-(3,5-Dihexyloxybenzyloxy)phenyl]acetylene (HGOPA). A mixture of 1-[4-(3,5-dihexyloxybenzyloxy)phenyl]2-trimethylsilylacetylene $(4.8 \mathrm{~g}, 10 \mathrm{mmol})$ and $\mathrm{K}_{2} \mathrm{CO}_{3}$ $(0.14 \mathrm{~g}, 1.0 \mathrm{mmol})$ in methanol $(200 \mathrm{~mL})$ was stirred at room temperature for $20 \mathrm{~h}$. After removal of the solvent, the residue was extracted with dichloromethane. The organic layer was washed with brine, then dried over anhydrous sodium sulfate, filtered, and evaporated. The crude product was purified by silica gel column separation with dichloromethane/hexane $(1 / 4 \mathrm{v} / \mathrm{v})$ as an eluent to give HG0PA $(3.5 \mathrm{~g}, 8.4 \mathrm{mmol})$. Yield $84 \%$. TLC (dichloromethane/hexane $(1 / 4 \mathrm{v} / \mathrm{v})): R_{f}=0.76$. IR $(\mathrm{KBr}$; $\left.\mathrm{cm}^{-1}\right): 3332$ (三C-H), 2860-2932 (C-H), $2160(\mathrm{C} \equiv \mathrm{C}) .{ }^{1} \mathrm{H}$ $\mathrm{NMR}\left(\mathrm{CDCl}_{3}, 270 \mathrm{MHz} ; \mathrm{ppm}\right): \delta 0.90(\mathrm{t}, 6 \mathrm{H}, J=6.8 \mathrm{~Hz}$, $\left.\mathrm{CH}_{3}\right), 1.33\left(\mathrm{~m}, 8 \mathrm{H}, \mathrm{CH}_{2}\right), 1.44\left(\mathrm{~m}, 4 \mathrm{H}, \mathrm{CH}_{2}\right), 1.76(\mathrm{~m}, 4 \mathrm{H}$, $\mathrm{CH}_{2}$ ), 2.99 ( $\left.\mathrm{s}, 1 \mathrm{H}, \mathrm{C} \equiv \mathrm{C}-\mathrm{H}\right), 3.93\left(\mathrm{t}, 4 \mathrm{H}, \mathrm{J}=6.6 \mathrm{~Hz}, \mathrm{CH}_{2} \mathrm{O}\right)$, 4.96 (s, 2H, $\left.\mathrm{CH}_{2} \mathrm{O}\right), 6.40(\mathrm{t}, 1 \mathrm{H}, \mathrm{J}=2.2 \mathrm{~Hz}, \mathrm{ArH}), 6.53$ (d, $2 \mathrm{H}, J=2.2 \mathrm{~Hz}, \mathrm{ArH}), 6.89(\mathrm{~d}, 2 \mathrm{H}, J=8.9 \mathrm{~Hz}, \mathrm{ArH}), 7.41$ $(\mathrm{d}, 2 \mathrm{H}, J=8.9 \mathrm{~Hz}, \mathrm{ArH}) .{ }^{13} \mathrm{C} \mathrm{NMR}\left(\mathrm{CDCl}_{3} ; \mathrm{ppm}\right): \delta 14.13$, 22.68, 25.79, 29.27, 31.64, 68.08, 70.04, 75.82, 83.60, 100.75, $105.53,114.31,114.77,133.47,138.57,158.92,160.39$.
4-Iodo-1-[3,5-bis(3,5-dihexyloxybenzyloxy)benzyloxy]benzene. A mixture of 4-iodophenol $(3.7 \mathrm{~g}, 17 \mathrm{mmol})$, 3,5-bis(3,5-dihexyloxybenzyloxy)benzyl chloride (13 g, $17 \mathrm{mmol}), \mathrm{K}_{2} \mathrm{CO}_{3}(4.7 \mathrm{~g}, 34 \mathrm{mmol})$, and 18-crown-6 $(0.56 \mathrm{~g}, 2.1 \mathrm{mmol})$ in acetone $(130 \mathrm{~mL})$ was refluxed for $115 \mathrm{~h}$. The mixture was worked up in the same manner as described above, and the crude product was purified by silica gel column separation with ethyl acetate/hexane $(1 / 8 \mathrm{v} / \mathrm{v})$ as an eluent to give 4-iodo-1-[3,5-bis(3,5dihexyloxybenzyloxy)benzyloxy]benzene (11 g, $12 \mathrm{mmol})$. Yield $72 \%$. TLC (ethyl acetate/hexane $(1 / 8 \mathrm{v} / \mathrm{v})): R_{f}=$ 0.64. IR (KBr; cm $\left.{ }^{-1}\right)$ : 2864-2936 (C-H). ${ }^{1} \mathrm{H} \mathrm{NMR}\left(\mathrm{CDCl}_{3}\right.$, $270 \mathrm{MHz} ; \mathrm{ppm}): \delta 0.90\left(\mathrm{t}, 12 \mathrm{H}, J=6.9 \mathrm{~Hz}, \mathrm{CH}_{3}\right), 1.33(\mathrm{~m}$, $\left.16 \mathrm{H}, \mathrm{CH}_{2}\right), 1.45\left(\mathrm{~m}, 8 \mathrm{H}, \mathrm{CH}_{2}\right), 1.76\left(\mathrm{~m}, 8 \mathrm{H}, \mathrm{CH}_{2}\right), 3.93(\mathrm{t}$, $\left.8 \mathrm{H}, \mathrm{J}=6.6 \mathrm{~Hz}, \mathrm{CH}_{2} \mathrm{O}\right), 4.94\left(\mathrm{~s}, 4 \mathrm{H}, \mathrm{CH}_{2} \mathrm{O}\right), 4.95(\mathrm{~s}, 2 \mathrm{H}$, $\left.\mathrm{CH}_{2} \mathrm{O}\right), 6.40(\mathrm{t}, 2 \mathrm{H}, \mathrm{J}=2.2 \mathrm{~Hz}, \mathrm{ArH}), 6.54(\mathrm{~d}, 4 \mathrm{H}, J=2.2 \mathrm{~Hz}$, $\operatorname{ArH}), 6.55(\mathrm{t}, 1 \mathrm{H}, J=2.2 \mathrm{~Hz}, \mathrm{ArH}), 6.62(\mathrm{~d}, 2 \mathrm{H}, J=2.2 \mathrm{~Hz}$, $\operatorname{ArH}), 6.71(\mathrm{~d}, 2 \mathrm{H}, J=9.0 \mathrm{~Hz}, \operatorname{ArH}), 7.53(\mathrm{~d}, 2 \mathrm{H}, J=9.0 \mathrm{~Hz}$, $\mathrm{ArH}) .{ }^{13} \mathrm{C} \mathrm{NMR}\left(\mathrm{CDCl}_{3} ; \mathrm{ppm}\right): \delta 14.15,22.69,25.80,29.28$, $31.64,68.05,69.93,70.13,83.07,100.69,101.50,105.62$, $106.11,117.20,138.07,138.70,138.75,158.31,159.97$, 160.32 .

1-\{4-[3,5-Bis(3,5-dihexyloxybenzyloxy)benzyloxy]phenyl\}2-trimethylsilylacetylene. Trimethylsilylacetylene $(1.4 \mathrm{~mL}$, $10 \mathrm{mmol})$ was added to a triethylamine solution $(10 \mathrm{~mL})$ of 4-iodo-1-[3,5-bis(3,5-dihexyloxybenzyloxy)benzyloxy] benzene ( $4.6 \mathrm{~g}, 5.0 \mathrm{mmol}$ ), bis(triphenylphosphine) palladium (II) chloride ( $10 \mathrm{mg}, 0.015 \mathrm{mmol})$, triphenylphosphine (4.2 $\mathrm{mg}, 0.016 \mathrm{mmol}$ ), and copper (I) iodide (17 mg, $0.090 \mathrm{mmol}$ ) under a nitrogen atmosphere. The solution was stirred for $14 \mathrm{~h}$ at $75^{\circ} \mathrm{C}$. The mixture was worked up in the same manner as described above, and the crude product was purified by silica gel column separation with dichloromethane/hexane $(1 / 2 \mathrm{v} / \mathrm{v})$ as an eluent to give 1- $\{4$-[3,5-bis(3,5-dihexyloxybenzyloxy)benzyloxy]phenyl $\}$ 2-trimethylsilylacetylene $(3.5 \mathrm{~g}, 4.0 \mathrm{mmol})$. Yield 79\%. IR $\left(\mathrm{KBr} ; \mathrm{cm}^{-1}\right)$ : $2864-2936(\mathrm{C}-\mathrm{H}), 2160(\mathrm{C} \equiv \mathrm{C}) .{ }^{1} \mathrm{H}$ NMR $\left(\mathrm{CDCl}_{3}, 500 \mathrm{MHz} ; \mathrm{ppm}\right): \delta 0.24\left(\mathrm{~s}, 9 \mathrm{H}, \mathrm{Si}\left(\mathrm{CH}_{3}\right)_{3}\right), 0.90(\mathrm{t}$, $\left.12 \mathrm{H}, J=7.0 \mathrm{~Hz}, \mathrm{CH}_{3}\right), 1.33\left(\mathrm{~m}, 16 \mathrm{H}, \mathrm{CH}_{2}\right), 1.45(\mathrm{~m}, 8 \mathrm{H}$, $\left.\mathrm{CH}_{2}\right), 1.76\left(\mathrm{~m}, 8 \mathrm{H}, \mathrm{CH}_{2}\right), 3.93\left(\mathrm{t}, 8 \mathrm{H}, \mathrm{J}=6.8 \mathrm{~Hz}, \mathrm{CH}_{2} \mathrm{O}\right)$, $4.94\left(\mathrm{~s}, 4 \mathrm{H}, \mathrm{CH}_{2} \mathrm{O}\right), 4.99\left(\mathrm{~s}, 2 \mathrm{H}, \mathrm{CH}_{2} \mathrm{O}\right), 6.40(\mathrm{t}, 2 \mathrm{H}, J=$ $2.2 \mathrm{~Hz}, \operatorname{ArH}), 6.54(\mathrm{~d}, 4 \mathrm{H}, J=2.2 \mathrm{~Hz}, \operatorname{ArH}), 6.56(\mathrm{t}, 1 \mathrm{H}, J$ $=2.2 \mathrm{~Hz}, \operatorname{ArH}), 6.63(\mathrm{~d}, 2 \mathrm{H}, J=2.2 \mathrm{~Hz}, \operatorname{ArH}), 6.86(\mathrm{~d}, 2 \mathrm{H}$, $J=9.0 \mathrm{~Hz}, \mathrm{ArH}), 7.38$ (d, $2 \mathrm{H}, J=9.0 \mathrm{~Hz}, \mathrm{ArH}) .{ }^{13} \mathrm{C} \mathrm{NMR}$ $\left(\mathrm{CDCl}_{3} ; \mathrm{ppm}\right): \delta 0.04,14.03,22.59,25.72,29.21,31.57$, $68.05,69.85,70.15,92.53,100.79,101.60,105.11,105.69$, $106.22,114.72,115.55,133.44,138.86,138.92,158.76$, $160.13,160.49$.

\{4-[3,5-Bis(3,5-dihexyloxybenzyloxy)benzyloxy]phenyl $\}$ acetylene (HG1PA). A mixture of 1-\{4-[3,5-bis(3,5-dihexyloxybenzyloxy)benzyloxy] phenyl $\}$-2-trimethylsilylacetylene $(3.5 \mathrm{~g}, 4.0 \mathrm{mmol})$ and $\mathrm{K}_{2} \mathrm{CO}_{3}(78 \mathrm{mg}, 0.57 \mathrm{mmol})$ in methanol/diethyl ether $(160 \mathrm{~mL} / 50 \mathrm{~mL})$ was stirred at room temperature for $24 \mathrm{~h}$. The mixture was worked up in the same manner as described above, and the crude product was purified by silica gel column separation 
with dichloromethane/hexane $(1 / 1 \mathrm{v} / \mathrm{v})$ as an eluent to give HG1PA $(2.3 \mathrm{~g}, 2.8 \mathrm{mmol})$. Yield $69 \%$. TLC (dichloromethane/hexane $(1 / 1 \mathrm{v} / \mathrm{v})): R_{f}=0.43 . \mathrm{IR}(\mathrm{KBr}$; $\left.\mathrm{cm}^{-1}\right): 3296(\equiv \mathrm{C}-\mathrm{H}), 2864-2940(\mathrm{C}-\mathrm{H}), 2112(\mathrm{C} \equiv \mathrm{C}) .{ }^{1} \mathrm{H}$ NMR $\left(\mathrm{CDCl}_{3}, 270 \mathrm{MHz} ; \mathrm{ppm}\right): \delta 0.90(\mathrm{t}, 12 \mathrm{H}, J=6.5 \mathrm{~Hz}$, $\left.\mathrm{CH}_{3}\right), 1.33\left(\mathrm{~m}, 16 \mathrm{H}, \mathrm{CH}_{2}\right), 1.45\left(\mathrm{~m}, 8 \mathrm{H}, \mathrm{CH}_{2}\right), 1.76(\mathrm{~m}, 8 \mathrm{H}$, $\left.\mathrm{CH}_{2}\right), 2.99(\mathrm{~s}, 1 \mathrm{H}, \mathrm{C} \equiv \mathrm{C}-\mathrm{H}), 3.93\left(\mathrm{t}, 8 \mathrm{H}, J=6.5 \mathrm{~Hz}, \mathrm{CH}_{2} \mathrm{O}\right)$, $4.94\left(\mathrm{~s}, 4 \mathrm{H}, \mathrm{CH}_{2} \mathrm{O}\right), 4.99\left(\mathrm{~s}, 2 \mathrm{H}, \mathrm{CH}_{2} \mathrm{O}\right), 6.40(\mathrm{t}, 2 \mathrm{H}, J=$ $2.2 \mathrm{~Hz}, \operatorname{ArH}), 6.54(\mathrm{~d}, 4 \mathrm{H}, J=2.2 \mathrm{~Hz}, \operatorname{ArH}), 6.56(\mathrm{t}, 1 \mathrm{H}, J$ $=2.2 \mathrm{~Hz}, \operatorname{ArH}), 6.64(\mathrm{~d}, 2 \mathrm{H}, J=2.2 \mathrm{~Hz}, \operatorname{ArH}), 6.88(\mathrm{~d}, 2 \mathrm{H}$, $J=8.8 \mathrm{~Hz}, \operatorname{ArH}), 7.41(\mathrm{~d}, 2 \mathrm{H}, J=8.8 \mathrm{~Hz}, \mathrm{ArH}) .{ }^{13} \mathrm{C} \mathrm{NMR}$ $\left(\mathrm{CDCl}_{3} ; \mathrm{ppm}\right): \delta 14.16,22.70,25.81,29.29,31.66,68.06$, $69.90,70.15,75.86,83.58,100.72,101.54,105.63,106.16$, $114.36,114.77,133.47,138.72,138.77,158.82,159.99$, 160.35 .

1-(3-Hydroxyl-3-methylbutynyl)-3,5-bis [(4-hexyloxyphenyl) ethynyl]benzene. A triethylamine $(40 \mathrm{~mL})$ solution of 4-[2(3,5-dibromophenyl)ethynyl]-1-(3-hydroxyl-3-methylbutynyl) benzene $(11 \mathrm{~g}, 35 \mathrm{mmol})$, Hex0H $(14 \mathrm{~g}, 70 \mathrm{mmol})$, bis(triphenylphosphine)palladium (II) chloride $(0.23 \mathrm{~g}$, $0.33 \mathrm{mmol})$, triphenylphosphine $(0.47 \mathrm{~g}, 1.8 \mathrm{mmol})$, and copper (I) iodide $(0.24 \mathrm{~g}, 1.3 \mathrm{mmol})$ was stirred for $16 \mathrm{~h}$ at $95^{\circ} \mathrm{C}$ under a nitrogen atmosphere. The mixture was worked up in the same manner as described above, and the crude product was purified by silica gel column separation with dichloromethane as an eluent to give 1-(3hydroxyl-3-methylbutynyl)-3,5-bis [(4-hexyloxyphenyl) ethynyl]benzene. (12 g, $21 \mathrm{mmol})$. Yield $60 \%$. TLC (dichloromethane): $R_{f}=0.34$. IR $\left(\mathrm{KBr} ; \mathrm{cm}^{-1}\right): 3464$ $(-\mathrm{OH}), 2960(\mathrm{C}-\mathrm{H}), 2212(\mathrm{C} \equiv \mathrm{C}) .{ }^{1} \mathrm{H}$ NMR $\left(\mathrm{CDCl}_{3}\right.$, $500 \mathrm{MHz} ; \mathrm{ppm}): \delta 0.91\left(\mathrm{t}, 6 \mathrm{H}, J=7.0 \mathrm{~Hz}, \mathrm{CH}_{3}\right), 1.35(\mathrm{~m}$, $\left.8 \mathrm{H}, \mathrm{CH}_{2}\right), 1.46\left(\mathrm{~m}, 4 \mathrm{H}, \mathrm{CH}_{2}\right), 1.62\left(\mathrm{~s}, 6 \mathrm{H}, \mathrm{CH}_{3}\right), 1.79(\mathrm{~m}$, $\left.4 \mathrm{H}, \mathrm{CH}_{2}\right), 2.01(\mathrm{~s}, 1 \mathrm{H}, \mathrm{OH}), 3.97\left(\mathrm{t}, 4 \mathrm{H}, J=6.7 \mathrm{~Hz}, \mathrm{CH}_{2} \mathrm{O}\right)$, $6.87(\mathrm{~d}, 4 \mathrm{H}, J=8.8 \mathrm{~Hz}, \operatorname{ArH}), 7.44(\mathrm{~d}, 4 \mathrm{H}, J=8.8 \mathrm{~Hz}$, $\operatorname{ArH}), 7.48(\mathrm{~d}, 2 \mathrm{H}, J=2.0 \mathrm{~Hz}, \operatorname{ArH}), 7.57(\mathrm{t}, 1 \mathrm{H}, J=2.0 \mathrm{~Hz}$, $\mathrm{ArH}) .{ }^{13} \mathrm{C}$ NMR $\left(\mathrm{CDCl}_{3} ; \mathrm{ppm}\right): \delta 14.03,22.59,25.68,29.14$, $31.41,31.56,65.58,68.10,80.87,86.52,90.59,94.62,114.58$, $123.28,124.25,133.11,133.35,133.58,133.74,159.46$.

\{3,5-Bis [(4-hexyloxyphenyl)ethynyl]phenyl\} acetylene (Hex1H). 1-(3-Hydroxyl-3-methylbutynyl)-3,5-bis [(4-hexyloxyphenyl)ethynyl]benzene $(12 \mathrm{~g}, 22 \mathrm{mmol}$ ) was allowed to react with sodium hydride in the same manner as described above. The crude product was purified by silica gel column separation with dichloromethane and hexane as an eluent to give $\mathbf{H e x} 1 \mathbf{H}(9.0 \mathrm{~g}, 18 \mathrm{mmol})$. Yield $82 \%$. TLC (dichloromethane): $R_{f}=0.93$. IR $\left(\mathrm{NaCl} ; \mathrm{cm}^{-1}\right): 3308$ $(\equiv \mathrm{C}-\mathrm{H}), 2932(\mathrm{C}-\mathrm{H}), 2208(\mathrm{C} \equiv \mathrm{C}) .{ }^{1} \mathrm{H} \mathrm{NMR}\left(\mathrm{CDCl}_{3}\right.$, $500 \mathrm{MHz} ; \mathrm{ppm}): \delta 0.91\left(\mathrm{t}, 6 \mathrm{H}, J=7.0 \mathrm{~Hz}, \mathrm{CH}_{3}\right), 1.35(\mathrm{~m}$, $\left.8 \mathrm{H}, \mathrm{CH}_{2}\right), 1.43\left(\mathrm{~m}, 4 \mathrm{H}, \mathrm{CH}_{2}\right), 1.78\left(\mathrm{~m}, 4 \mathrm{H}, \mathrm{CH}_{2}\right), 3.09(\mathrm{~s}$, $1 \mathrm{H}, \mathrm{C} \equiv \mathrm{C}-\mathrm{H}), 3.97\left(\mathrm{t}, 4 \mathrm{H}, J=6.8 \mathrm{~Hz}, \mathrm{CH}_{2} \mathrm{O}\right), 6.87(\mathrm{~d}, 4 \mathrm{H}, J$ $=9.0 \mathrm{~Hz}, \mathrm{ArH}), 7.44(\mathrm{~d}, 4 \mathrm{H}, J=9.0 \mathrm{~Hz}, \mathrm{ArH}), 7.55(\mathrm{~d}, 2 \mathrm{H}$, $J=1.5 \mathrm{~Hz}, \mathrm{ArH}), 7.62(\mathrm{t}, 1 \mathrm{H}, J=1.5 \mathrm{~Hz}, \mathrm{ArH}) .{ }^{13} \mathrm{C} \mathrm{NMR}$ $\left(\mathrm{CDCl}_{3} ; \mathrm{ppm}\right): \delta 14.03,22.59,25.69,29.14,31.57,68.11$, $78.03,82.21,86.39,90.75,114.52,114.59,122.68,124.37$, $133.14,133.99,134.31,159.51$.
2.3. Polymerization. An appropriate amount of monomers (typically, $0.5-1.0 \mathrm{~g}$ ) was placed in a Schlenk tube equipped with a three-way stopcock, a rubber septum, and a Tefloncoated magnetic stirring bar. The tube was placed under vacuum, followed by a nitrogen backflush. Freshly distilled solvent was transferred to the tube, and the monomers were dissolved with stirring. The determined amount of $[\mathrm{Rh}(\mathrm{nbd}) \mathrm{Cl}]_{2}$ and triethylamine dissolved in the solvent was added to the stirred monomers solution. The detailed polymerization conditions are tabulated in Table 1 . The reaction solution was poured into methanol or methanol/benzene $(3 / 2 \mathrm{v} / \mathrm{v})$ to yield polymer precipitate. The precipitate was washed with the precipitant and then dried in vacuo to give a yellow polymer.

2.4. Membrane Preparation. A $5 \mathrm{wt} \%(\mathrm{w} / \mathrm{v})$ solution of a polymer in chloroform or toluene was cast on a Teflon sheet, and the solvent was evaporated at room temperature. The resulting solid membrane was detached from the sheet and dried in vacuo for $24 \mathrm{hr}$. Thickness (L) of the membranes was $70-160 \mu \mathrm{m}$.

2.5. Measurement of Oxygen and Nitrogen Permeability. Oxygen and nitrogen permeability coefficients $\left(\mathrm{P}_{\mathrm{O}_{2}}\right.$ and $\mathrm{P}_{\mathrm{N}_{2}}$ : $\mathrm{cm}^{3}$ (STP) $\mathrm{cm} \mathrm{cm}^{-2} \mathrm{~s}^{-1} \mathrm{cmHg}^{-1}$ ) and the oxygen separation factor $\left(\alpha=\mathrm{P}_{\mathrm{O}_{2}} / \mathrm{P}_{\mathrm{N}_{2}}\right)$ were measured by a gas chromatographic method using YANACO GTR-10 according to [13].

2.6. Other Measurements. IR spectra were measured with a Hitachi IR 270-30 spectrometer. NMR $\left({ }^{1} \mathrm{H},{ }^{13} \mathrm{C}\right)$ spectra were measured with a Varian Unity $500 \mathrm{SW}(500 \mathrm{MHz})$ or a JEOL GSX-270 $(270 \mathrm{MHz})$ spectrometer. Average molecular weights $\left(M_{\mathrm{n}}\right.$ and $\left.M_{\mathrm{w}}\right)$ were evaluated by coupling of gel permeation chromatography and low angle laser light scattering (GPC-LALLS) at $40^{\circ} \mathrm{C}$ on THF eluent using Tosoh Liquid Chromatograph instruments with SD-8000, CCPD, C0-8010, LS-8000, RI-8011, and PP8010. The optical spectra were measured with a JASCO Ubest V-550DS UV-vis spectrometer. The wide-angle X-ray scattering measurements were performed using a Rigaku Geigerflex with a graphitemonochromatized $\mathrm{Cu} \mathrm{K} \alpha$ radiation, which was supplied at $40 \mathrm{kV}$ and $20 \mathrm{~mA}$.

\section{Results and Discussion}

3.1. Polymerization of Monodendron Monomers. The monodendron monomers were polymerized by $\mathrm{Rh}$ catalyst, $[\mathrm{Rh}(\mathrm{nbd}) \mathrm{Cl}]_{2}$, in the presence of triethylamine cocatalyst. The polymerization mixtures were purified by precipitating into methanol or methanol/benzene $(9 / 1-4 / 3 \mathrm{v} / \mathrm{v})$ to yield the corresponding polydendrons as yellow powders. It is well known that the $\mathrm{Rh}$ catalyst can selectively promote the polymerization of monosubstituted acetylenes [14-19]. Moreover, we have previously demonstrated that TMS1H, PMDS1H, and NMTS1H polymerize at the terminal acetylene group of the focal point [7]. The same catalyst system promotes the polymerization of HG1PA and $\mathrm{Hex} 1 \mathrm{H}$ to yield the corresponding polymers as shown in Table 1. HG0PA 
TABLE 1: Polymerization of phenylacetylene monodendrons with alkyl peripheral groups using $[\mathrm{Rh}(\mathrm{nbd}) \mathrm{CL}]_{2}{ }^{\mathrm{a}}$.

\begin{tabular}{lcccc}
\hline Monomers & Yield $(\%)$ & $\begin{array}{c}M_{\mathrm{w}}^{\mathrm{b}} \\
\left(\times 10^{6}\right)\end{array}$ & $M_{\mathrm{w}} / M_{\mathrm{n}}^{\mathrm{b}}$ & $\begin{array}{c}D P^{\mathrm{c}} \\
\left(\times 10^{3}\right)\end{array}$ \\
\hline MG0PA & 60 & 0.92 & 1.4 & 3.8 \\
HG0PA $^{\mathrm{d}}$ & 87 & 2.4 & 2.7 & 5.9 \\
DG0PA $^{\mathrm{e}}$ & 65 & 2.8 & 1.7 & 7.9 \\
HG1PA $_{\text {Hex0H }}^{\mathrm{f}}$ & 92 & 6.1 & $5.2^{\mathrm{g}}$ & \\
Hex1H $^{\mathrm{f}}$ & 49 & $5.3^{\mathrm{g}}$ & $3.0^{\mathrm{g}}$ & \\
\hline
\end{tabular}

${ }^{a}$ Triethylamine, $[M]_{0}=0.5 \mathrm{~mol} / \mathrm{l},[\mathrm{M}]_{0} /[\mathrm{Cat}]_{0}=2500,25^{\circ} \mathrm{C}, 3 \mathrm{hr}$, precipitated with $\mathrm{MeOH} / \mathrm{benzene}(9 / 1 \mathrm{v} / \mathrm{v})$.

${ }^{\mathrm{b}}$ Determined from GPC-LALLS.

${ }^{c}$ Calculated from $M_{\mathrm{w}}$.

${ }^{\mathrm{d}}$ Ether, [triethylamine $]_{0} /[\text { Cat }]_{0}=20$.

e $[M]_{0} /[\text { Cat }]_{0}=5000$.

${ }^{\mathrm{f}}$ Chloroform, $[M]_{0} /[\text { Cat }]_{0}=1000$, $[\text { triethylamine }]_{0} /[\text { Cat }]_{0}=20$.

$\mathrm{g}$ Determined from GPC calibrated by polystyrene standard.

and DGOPA gave highly soluble polymers with a high degree of polymerization $\left(D P>10^{3}\right)$ in spite of the larger peripheral groups in comparison with MG0PA. The yield and the degree of polymerization of polydendrons (i.e., poly(HG1PA) and poly $(\mathbf{H e x} 1 \mathbf{H}))$ are similar to those of the corresponding zerogeneration polymers (i.e., $\operatorname{poly}(\mathrm{HGOPA})$ and $\operatorname{poly}(\mathrm{Hex} 1 \mathrm{H})$ ), since the first-generation dendrons are not too crowded to affect the polymerization ability.

3.2. Structure of Polydendrons. The polymerization of the monodendrons at the terminal acetylene group of focal point was confirmed by means of IR and ${ }^{1} \mathrm{H}$ NMR; for example, in IR and ${ }^{1} \mathrm{H}$ NMR spectra of $\operatorname{poly}(\operatorname{Hex} 1 \mathbf{H})$, the peaks assignable to the terminal acetylene group of the monodendron $\mathrm{Hex} 1 \mathrm{H}$, that is, $3308 \mathrm{~cm}^{-1}$ (the stretching vibration of the $\equiv \mathrm{C}-\mathrm{H}$ bond) in IR spectrum and $\delta 3.09$ (s, $1 \mathrm{H}, \equiv \mathrm{C}-\mathrm{H})$ in ${ }^{1} \mathrm{H}$ NMR, completely disappeared. The visible absorption maxima $\left(\lambda_{\max }\right)$ of polydendrons significantly increased due to the construction of the dendritic structure, that is, poly(Hex0H) $(400 \mathrm{~nm})$ to $\operatorname{poly}(\mathbf{H e x} \mathbf{H})(455 \mathrm{~nm})$, and poly(HG0PA) (400 nm) to poly(HG1PA) (441 nm). A bathochromic shift has been reported for some orthoand meta-substituted poly(phenylacetylene)s in comparison with the simple or para-substituted poly(phenylacetylene) [20-24]. This bathochromic shift indicates a developed $\pi$-conjugation in the main chain of polydendrons and suggests that steric hindrance and repulsion among bulky substituents at the $m$-position formed the extended main chain structure. This resulted in a highly twisted dihedral angle between the main chain and the attached phenyl rings and was counteracted by slightly or moderately twisted single bonds of the main chain. The visible absorption maxima $\left(\lambda_{\max }\right)$ of the first-generation polydendrons were significantly increased due to the effect of the bulky substituent at the $m$-position in comparison with the corresponding zerogeneration polymers poly(HGOPA) and poly(Hex1H).

The wide-angle X-ray scattering (WAXS) of poly(HG0PA) and poly(HG1PA) was measured in film states (Table 2). The sharp peaks were observed at $2 \theta=$ $3-4^{\circ}$, and these sharp crystalline peaks are attributed to the (100) reflection of the pseudohexagonal lattice of rod-like molecules $[7,25]$. The interplanar $d$ spacing of the polymers increased with increasing generation, while the density seldom decreased. These data suggest that the increase of $d$ spacing is related to the increase of the column diameter with the generation. The column diameter of the polymers estimated from $d$ spacing is only slightly smaller than that of molecular modeling. (The molecular modeling was performed on the assumption of a fully extended conformation for side-chain monodendrons and distorted cis-transoid main chain conformation (dihedral angle $=130 \mathrm{deg}$ ), as described in [7].) The column diameter difference between the values estimated from $d$ spacing and from molecular modeling suggests that the flexible alkyl chain as the peripheral group was bent and/or the columns slightly overlapped each other in the part of the peripheral group.

3.3. Oxygen Permselectivity of Polydendron Membranes. The polymers were soluble in common organic solvents, such as chloroform, toluene, and tetrahydrofuran. The polymers poly(HG0PA), poly(Hex0H), and poly (Hex1H) exhibited good film-forming abilities depending on their degree of polymerization and formed a self-supporting film colored orange due to $\pi$-conjugated main chain chromophore by the solvent-casting method.

Oxygen and nitrogen permeation were measured using air as feed gas at $298 \mathrm{~K}$ and $76 \mathrm{cmHg}$ using poly(HG0PA), poly $(\mathbf{H e x} \mathbf{0 H})$, and poly $(\mathbf{H e x} \mathbf{1 H})$ membranes. Figure 1 shows the relationship between the oxygen permeability coefficient $\left(\mathrm{P}_{\mathrm{O}_{2}}\right)$ and the oxygen separation factor $\left(\alpha=\mathrm{P}_{\mathrm{O}_{2}} / \mathrm{P}_{\mathrm{N}_{2}}\right)$ for the polydendron membranes with the zero-generation polymer (poly(TMSOH), poly(PMDSOH), and poly(NMTSOH)) membranes. The oxygen permeability coefficient $\left(\mathrm{P}_{\mathrm{O}_{2}}\right)$ of the membranes formed by first-generation polydendrons were smaller than that of the corresponding zerogeneration poly(phenylacetylene) derivative. It seems that this behavior was caused by the low mobility of the stiff and crowded dendritic structure. The zero-generation poly(phenylacetylene) derivatives show a balance between 
TABLE 2: Column structure of polydendrons.

\begin{tabular}{lcccccc}
\hline Polymers & $\begin{array}{l}\text { Generation } \\
\text { number }\end{array}$ & $\begin{array}{c}\text { Density } \\
\left(\mathrm{g} / \mathrm{cm}^{3}\right)\end{array}$ & $2 \theta^{\mathrm{b}}(\mathrm{deg})$ & \multicolumn{2}{c}{$\begin{array}{c}d \text { spacing } \\
(\AA)\end{array}$} & \multicolumn{2}{c}{ Column diameter $^{\mathrm{c}}$} & WAXS $^{\mathrm{d}}(\AA)$ & Calcd $^{\mathrm{e}}(\AA)$ \\
\hline poly(HG1PA $)^{\mathrm{f}}$ & 1 & 1.068 & 3.1 & 30.5 & 35.2 & 43.0 \\
poly(HG0PA $)^{\mathrm{f}}$ & 0 & 1.062 & 3.3 & 28.7 & 33.1 & 36.7 \\
\hline
\end{tabular}

${ }^{a}$ Determined by floating method containing error of \pm 0.005 .

${ }^{\mathrm{b}}$ Crystalline peak of the wide-angle X-ray scattering (WAXS) of the polymers.

${ }^{\mathrm{c}}$ Estimated from $2 \theta$.

d Estimated from $d($ column diameter $=2 d / \sqrt{ } 3)$.

e Estimated from molecular modeling, which was performed as described in text. (The molecular modeling was performed on the assumption of a fully extended conformation for side-chain monodendronsn and distorted cis-transoid main chain conformation (dihedral angle $=130$ deg), as described in [7].)

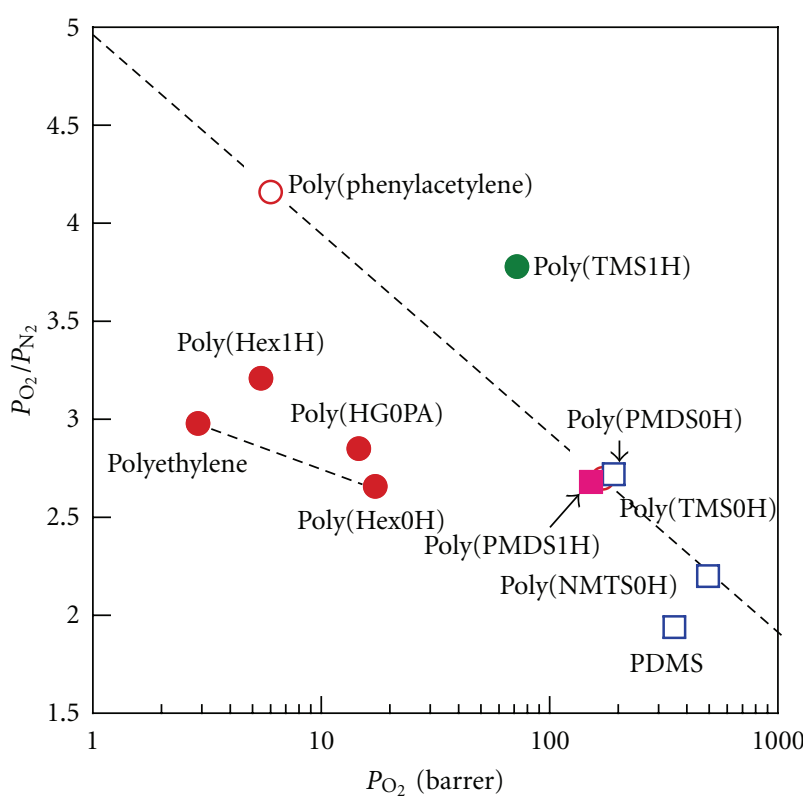

Figure 1: Permselectivity of poly(phenylacetylene) analogue membranes for $\mathrm{O}_{2} / \mathrm{N}_{2}$ separation. Poly(phenylacetylene) (PPA) and poly(TMSOH) from [13], poly(PMDSOH), poly(NMTSOH), poly(TMS1H), and poly(PMDS1H) from [6].

the oxygen permeability coefficient and oxygen separation factor. However, the oxygen permselectivity of the polydendrons with a trimethylsilyl group, poly(TMS1H), shows good performance in comparison with corresponding zerogeneration poly(phenylacetylene) derivatives. In comparison, polydendrons with oligo(dimethylsiloxane) peripheral groups (i.e., poly(PMDS1H)) exhibited similar trend to that of the corresponding zero-generation poly(phenylacetylene) derivatives. On the other hand, polymers and polydendrons with alkoxy peripheral groups exhibited the lower oxygen permeability coefficient due to the alkyl domain that would have better gas barrier property than oligo(dimethylsiloxane) domain. But the oxygen permselectivity of poly $(\mathbf{H e x} 1 \mathbf{H})$ shows good performance in comparison with the trend between polyethylene and poly $(\mathbf{H e x} \mathbf{0 H})$. These results suggest that the space-persistency due to the stiff and crowded dendritic structure play an important role in the high performance of the oxygen permselectivity, while the effect of the peripheral group was similar to that of the corresponding zero-generation poly(phenylacetylene) derivatives.

\section{Conclusions}

The phenylacetylene monomers consisting of monodendron with alkoxy peripheral groups were successfully polymerized with a $\mathrm{Rh}$ catalyst, $[\mathrm{Rh}(\mathrm{nbd}) \mathrm{Cl}]_{2}$, to yield corresponding polydendrons with high molecular weight, in spite of the bulkiness of the dendritic residue and the peripheral groups. The polydendrons were fabricated to self-supporting membranes, and the membrane formed by the polydendron of first generation, poly $(\mathbf{H e x} 1 \mathbf{H})$, showed an oxygen permselectivity higher than that of the corresponding zerogeneration poly(phenylacetylene) derivatives.

\section{Acknowledgments}

This work was partially supported by a Grant-in-Aid for Scientific Research (B) (no. 20310052) from JSPS and by the Mukai Science Technology Foundation.

\section{References}

[1] K. Inoue, "Functional dendrimers, hyperbranched and star polymers," Progress in Polymer Science, vol. 25, no. 4, pp. 453571, 2000.

[2] D. A. Tomalia and J. M. J. Fréchet, "Discovery of dendrimers and dendritic polymers: a brief historical perspective," Journal of Polymer Science Part A, vol. 40, no. 16, pp. 2719-2728, 2002.

[3] J. M. J. Fréchet, "Dendrimers and other dendritic macromolecules: from building blocks to functional assemblies in nanoscience and nanotechnology," Journal of Polymer Science Part A, vol. 41, no. 23, pp. 3713-3725, 2003.

[4] T. Aoki and T. Kaneko, "New macromolecular architectures for permselective membranes-gas permselective membranes from dendrimers and enantioselectively permeable membranes from one-handed helical polymers," Polymer Journal, vol. 37, no. 10, pp. 717-735, 2005.

[5] T. Kaneko, T. Horie, M. Asano, T. Aoki, and E. Oikawa, "Polydendron: polymerization of dendritic phenylacetylene monomers," Macromolecules, vol. 30, no. 10, pp. 3118-3121, 1997.

[6] T. Kaneko, K. Yamamoto, M. Asano, M. Teraguchi, and T. Aoki, "Synthesis of poly(phenylacetylene)-based polydendrons consisting of a phenyleneethynylene repeating unit, and oxygen/nitrogen permeation behavior of their membranes," Journal of Membrane Science, vol. 278, no. 1-2, pp. 365-372, 2006.

[7] T. Kaneko, M. Asano, K. Yamamoto, and T. Aoki, "Polymerization of phenylacetylene-based monodendrons and structure of 
the corresponding polydendrons," Polymer Journal, vol. 33, no. 11 , pp. 879-890, 2001.

[8] G. Chen, W. Shan, Y. Wu, L. Ren, J. Dong, and Z. Ji, "Synthesis and anti-inflammatory activity of resveratrol analogs," Chemical and Pharmaceutical Bulletin, vol. 53, no. 12, pp. 1587-1590, 2005.

[9] K. Sivanandan, S. V. Aathimanikandan, C. G. Arges, C. J. Bardeen, and S. Thayumanavan, "Probing every layer in dendrons," Journal of the American Chemical Society, vol. 127, no. 7, pp. 2020-2021, 2005.

[10] J. F. Eckert, J. F. Nicoud, J. F. Nierengarten et al., "Fullereneoligophenylenevinylene hybrids: synthesis, electronic properties, and incorporation in photovoltaic devices," Journal of the American Chemical Society, vol. 122, no. 31, pp. 7467-7479, 2000.

[11] O. Haba, K. Haga, M. Ueda, O. Morikawa, and H. Konishi, "A new photoresist based on calix[4] resorcinarene dendrimer," Chemistry of Materials, vol. 11, no. 2, pp. 427-432, 1999.

[12] S. Gandon, P. Mison, M. Bartholin et al., "Thermal polymerization of arylacetylenes: 1 . Study of a monofunctional model compound," Polymer, vol. 38, no. 6, pp. 1439-1447, 1997.

[13] T. Aoki, H. Nakahara, Y. Hayakawa, M. Kokai, and E. Oikawa, "Trimethylsilyl-group containing polyphenylacetylenes for oxygen and ethanol permselective membranes," Journal of Polymer Science Part A, vol. 32, no. 5, pp. 849-858, 1994.

[14] M. Tabata, W. Yang, and K. Yokota, "Polymerization of mchlorophenylacetylene initiated by [Rh(norbornadiene) CI]," Polymer Journal, vol. 22, no. 12, pp. 1105-1107, 1990.

[15] M. Tabata, W. Yang, and K. Yokota, "H-NMR and UV studies of Rh complexes as a stereoregular polymerization catalysts for phenylacetylenes: effects of ligands and solvents on its catalyst activity," Journal of Polymer Science Part A, vol. 32, no. 6, pp. 1113-1120, 1994.

[16] Y. Kishimoto, P. Eckerle, T. Miyatake et al., "Well-controlled polymerization of phenylacetylenes with organorhodium(I) complexes: mechanism and structure of the polyenes," Journal of the American Chemical Society, vol. 121, no. 51, pp. 1203512044, 1999.

[17] Y. Misumi and T. Masuda, "Living polymerization of phenylacetylene by novel rhodium catalysts. Quantitative initiation and introduction of functional groups at the initiating chain end," Macromolecules, vol. 31, no. 21, pp. 7572-7573, 1998.

[18] Y. Misumi, K. Kanki, M. Miyake, and T. Masuda, "Living polymerization of phenylacetylene by rhodium-based ternary catalysts, (diene)Rh(I) complex/vinyllithium/phosphorus ligand. Effects of catalyst components," Macromolecular Chemistry and Physics, vol. 201, no. 17, pp. 2239-2244, 2000.

[19] M. Miyake, Y. Misumi, and T. Masuda, "Living polymerization of phenylacetylene by isolated rhodium complexes," Macromolecules, vol. 33, no. 18, pp. 6636-6639, 2000.

[20] T. Masuda and T. Higashimura, "Polyacetylenes with substituents: their synthesis and properties," Advances in Polymer Science, vol. 81, pp. 121-165, 1986.

[21] J. Kunzler and V. Percec, "Living polymerization of aryl substituted acetylenes by MoCl," Journal of Polymer Science Part A, vol. 28, no. 5, pp. 1221-1236, 1990.

[22] H. Nishide, T. Kaneko, R. Gotoh, and E. Tsuchida, "Polyacetylene derivatives with chain-sided phenoxy and galvinoxyl radicals," Molecular Crystals and Liquid Crystals, vol. 233, pp. 89-96, 1993.

[23] Y. Miura, M. Matsumoto, and Y. Ushitani, "Synthesis of poly(ethynylbenzene) with pendant nitroxide radicals by rhodium-catalyzed polymerization of ethynylphenyl nitroxide," Macromolecules, vol. 26, no. 10, pp. 2628-2630, 1993.
[24] R. R. Schrock, S. Luo, J. C. Lee, N. C. Zanetti, and W. M. Davis, "Living polymerization of (o-(trimethylsilyl)phenyl)acetylene by molybdenum imido alkylidene complexes," Journal of the American Chemical Society, vol. 118, no. 16, pp. 3883-3895, 1996.

[25] M. Tabata, H. Takamura, K. Yokota et al., "Pressure-induced cis to trans isomerization of poly(o-methoxyphenylacetylene) polymerized by $\mathrm{Rh}$ complex catalyst. A ramam, X-ray, and ESR study," Macromolecules, vol. 27, no. 21, pp. 6234-6236, 1994. 

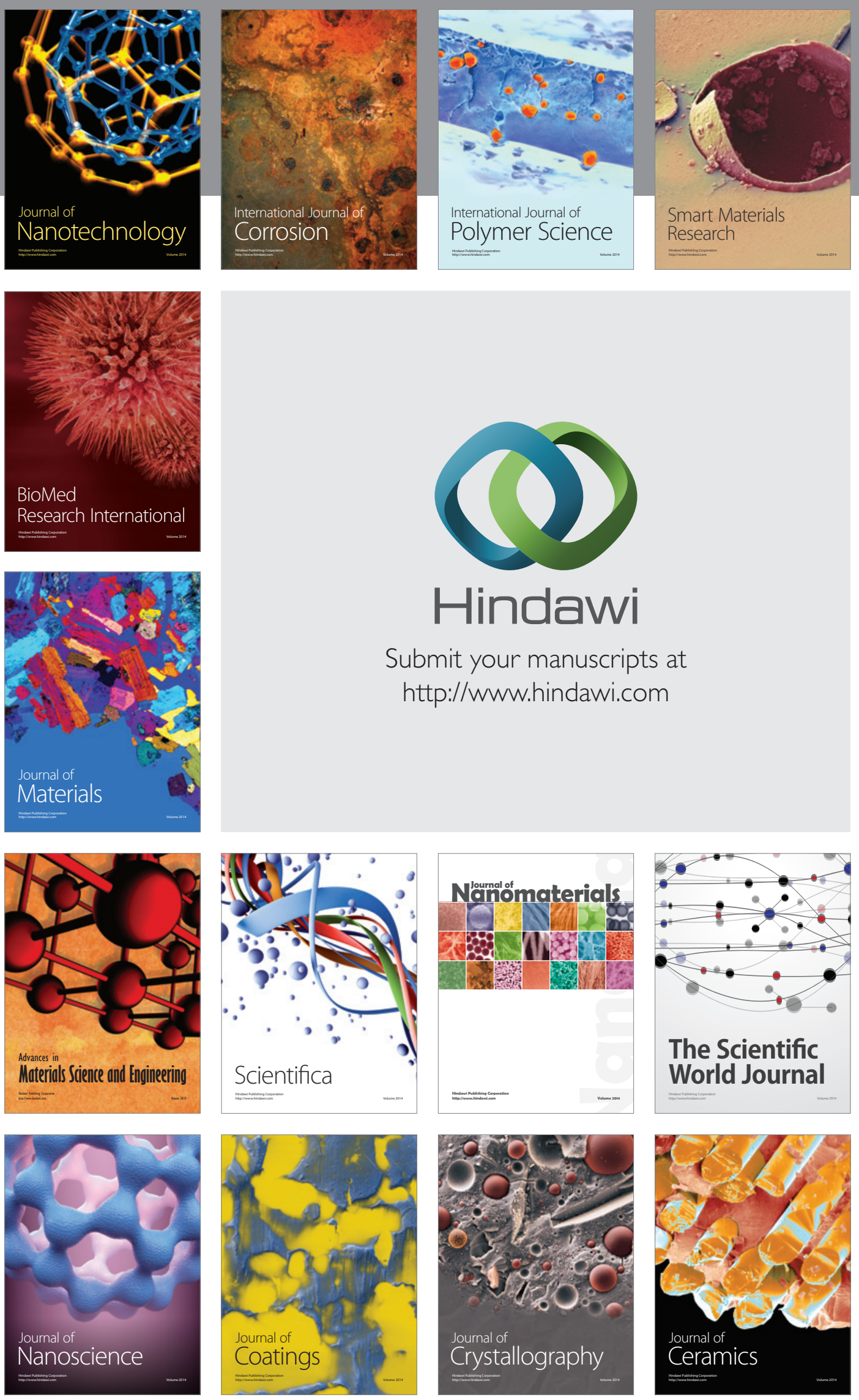

The Scientific World Journal

Submit your manuscripts at

http://www.hindawi.com

\section{World Journal}

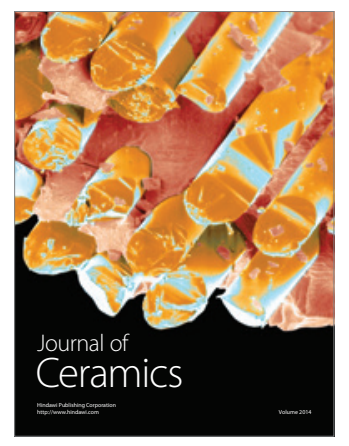

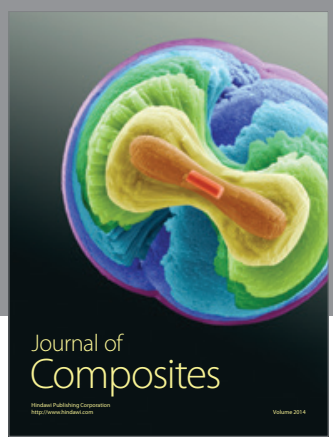
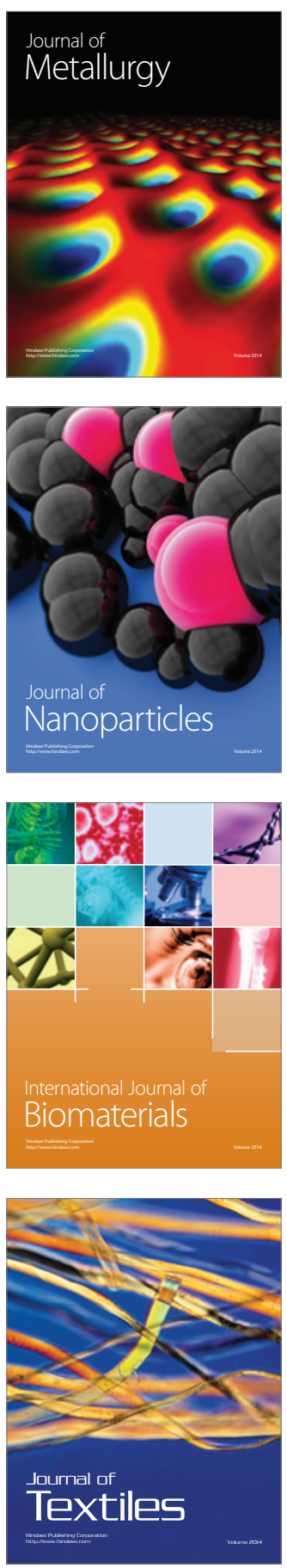